\title{
Stray light control and analysis for an off-axis three-mirror anastigmat telescope
}

\author{
Lionel Clermont ${ }^{\mathrm{a}, *}$ and Ludovic Aballea ${ }^{\mathrm{b}}$ \\ ${ }^{a}$ Université de Liège, STAR Institute, Centre Spatial de Liège, Liège, Belgium \\ ${ }^{\mathrm{b}}$ OIP Sensor Systems, Oudenaarde, Belgium
}

\begin{abstract}
Off-axis three-mirror anastigmat (TMA) telescopes provide excellent correction of aberrations over a large field-of-view in one direction. In a push broom configuration, this lightweight and compact optical configuration enables high-performing imaging. Moreover, passive multi-spectral acquisition can be achieved using a filter stack positioned at the detector vicinity. Stray light is a typical limiting factor for instrument performance and its control is specific to the type of optical configuration. We describe the stray light control and analysis methods in an offaxis TMA. The design intends to control first-order scattering from non-optical surfaces and to block straight shots. This is achieved through usage of elements such as apertures and baffles, both internal and external to the instrument. It will be demonstrated that the aperture stop is a critical element, whose stray light contribution can be controlled with V-groove vanes. Impact of mirrors roughness, a manufacturing physical limitation, is evaluated by modeling the bidirectional scattering distribution function and computing the stray light distribution at the detector for point-like source illumination. We show that the scattering on the different mirrors broaden differently the spot size, as the successive mirrors focus or expand the scattered rays differently. Finally, the ghost reflections inside the filter stack are evaluated and we show that there is no cross-talk between the different channels. (C) 2021 Society of Photo-Optical Instrumentation Engineers (SPIE) [DOI: 10.1117/1.OE.60.5.055106]
\end{abstract}

Keywords: stray light; three-mirror anastigmat; ghost reflection; scattering; ray tracing.

Paper 20201114SS received Sep. 20, 2020; accepted for publication May 12, 2021; published online May 28, 2021.

\section{Introduction}

An off-axis three-mirror anastigmat (TMA) is a type of telescope consisting of three curved mirrors arranged in an off-axis configuration. ${ }^{1}$ They enable an excellent correction of aberrations while being obscuration-free. ${ }^{1,2}$ Compact and lightweight TMAs are particularly interesting for Earth observation applications where space segment dimensions is becoming a constrain. While the field-of-view (FOV) along the off-axis is limited, TMAs are optimized with a large FOV in the perpendicular direction. ${ }^{1-3}$ In a push broom configuration, the large FOV scans the Earth in the across-track (ACT) direction. Along-track (ALT), the instrument looks at the same area of the Earth with a very small temporal shift. A detector with a stack of stripe spectral filters can be placed at the focal plane (FPA) to enable passive multi-spectral observation of the Earth. In that case, there is only a small temporal shift between the spectral bands. With the progresses of diamond turning technology, high-performance TMAs can now be built to provide wide coverage for Earth observation on smaller and lighter platforms than traditional on-axis refractive telescopes. ${ }^{4,5}$ For the future of Earth observation, TMAs are excellent candidates to fulfill a need for compact high-performing instruments.

In addition of aberrations correction, stray light is an important concern for optical instruments. Stray light designates any light that reaches the detector through a path or process different than the one intended by design. ${ }^{6}$ Its consequences can be the addition on the image of unwanted signal and features as well as the broadening of the point spread function (PSF). Stray light can occur due to ghost reflections or scattering on either optical or non-optical

*Address all correspondence to Lionel Clermont, lionel.clermont@uliege.be

0091-3286/2021/\$28.00 (C) 2021 SPIE

Optical Engineering

055106-1

May 2021 • Vol. 60(5) 
surfaces. In the case of off-axis reflection systems, straight shots can create intense stray light when rays are able to reach the detector without following the natural sequence of the optical elements. ${ }^{7}$ This can be very damaging as their power is not attenuated by a ghost or scattering event. Earth observation is a domain where stray light requirements are often particularly tight. Moreover, it is usually harder to achieve such requirements on instruments with large FOV. While there is not a unique and universal recipe for stray light control, general best practices are followed to ensure that the system has a sufficiently low stray light. This is done through smart opto-mechanical design and proper material selection, including black treatments. ${ }^{6,8}$ For example, stray light from non-optical surfaces is controlled by emphasizing on first-order scattering (i.e., light scattered only once before reaching the detector). These paths can be avoided using apertures, baffles, or vanes to prevent surfaces from being simultaneously illuminated and visible from the detector. ${ }^{8}$ Straight shots can also be blocked with this kind of element or with housing enclosure. ${ }^{7}$ Scattering from optical surfaces is minimized with clean and polished surfaces $;{ }^{6}$ however, there is obviously a physical limit. Regarding ghosts, these can be decreased by proper orientation of the optical surfaces with respect to each other, using anti-reflection (AR) coatings on refractive elements or even by optimizing the lenses curvatures so that the ghosts are either located outside of the photosensitive area or widespread at the detector, ${ }^{9}$ therefore presenting a smaller irradiance on each pixel. Stray light analysis is conducted to guide optomechanical design but also to predict and verify the design performances. For that purpose, an opto-mechanical model is built in a ray tracing software and the interactions with rays are computed to predict the stray light level at the detector. ${ }^{10,11}$ The typical approach is to ensure appropriate stray light performances at design level before initiating manufacturing. Indeed, correcting physically stray light on an already manufactured instrument is hard, if not impossible, and increases the complexity of calibration and stray light removal algorithms used for ground data processing.

In some kinds of design, for example, Cassegrain telescopes, ${ }^{6,8}$ the stray light control methods are well known and have been widely used. In this paper, we intend to describe the stray light control and analysis methods in the case of an off-axis TMA. Figure 1 shows the optical layout of the system considered here, where a global reference frame $X Y Z$ is defined. The system is designed by OIP Sensor Systems, which had also developed previously the ProbaV instrument in a TMA configuration. It consists of three curved mirrors with the aperture stop on the secondary and an off-axis along $Y Z$. The instrument is quasi-telecentric with an effective focal length (EFL) of $290.971 \mathrm{~mm}$ and root mean square (RMS) spot size between 2 and $6 \mu \mathrm{m}$. It has an FOV of about $\pm 7.2 \mathrm{deg}$ in the ACT direction, aligned with the $X$ axis. In the ALT direction, along the $Y$ axis, the spectral channels are contained within a full FOV of $2.4 \mathrm{deg}$. A fold mirror is placed after M3 to separate the spectral bands on two different FPAs. The advantage is that it allows to use two different types of detectors with potentially very different

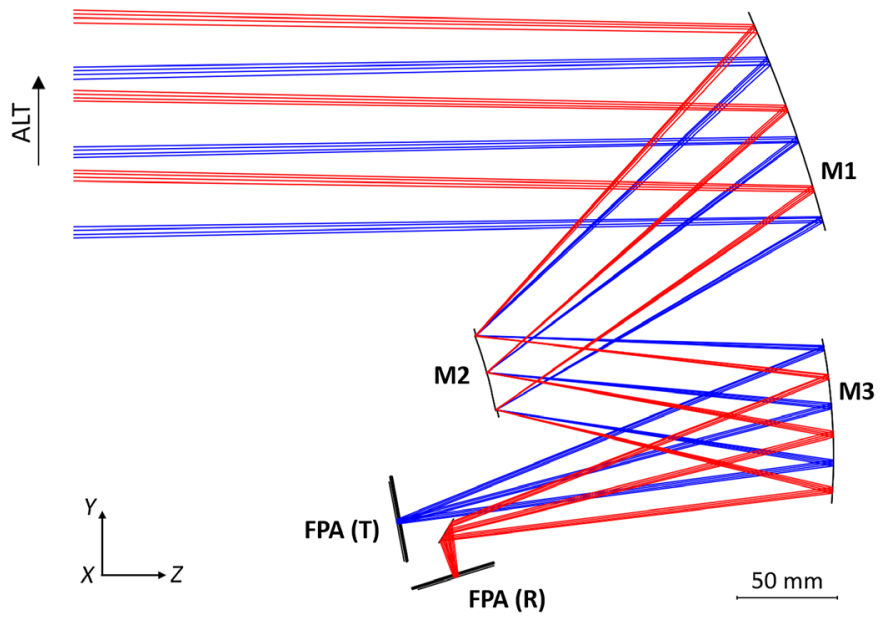

Fig. 1 Optical layout of an off-axis TMA. EFL $=290.971 \mathrm{~mm}$; full FOV 14.4 deg along $X$ (ACT) and 2.4 deg along $Y($ ALT). 


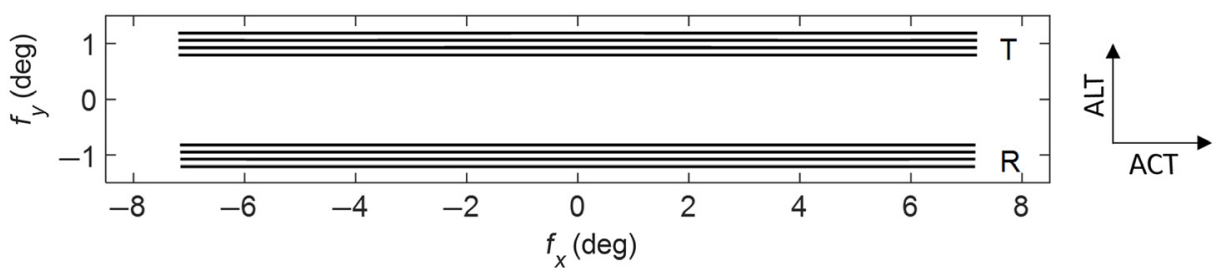

Fig. 2 FOV for the transmission (T) and reflection (R) channels of the TMA.

properties. The two FPAs are labeled $\mathrm{T}$ and $\mathrm{R}$, respectively, for transmission and reflection channels. Each FPA has a 4-line sensor, in front of which is placed a butcher block. The butcher block is a spectral filter window, which consists of an assembly of different spectral filters each composed of a stack of fused silica substrates with different dielectric coatings. The sensors have a semi-width of $39 \mathrm{~mm}$ and pixels of $13 \mu \mathrm{m}$. Table 3 gives the spot positions $\left(x_{0} ; y_{0}\right)$ and fields $\left(f_{x} ; f_{y}\right)$ for the center and edge of each channel. The spot position is given in a local reference frame with the $y$ axis aligned with the FPA spectral direction. The fields of the different channels are considered with respect to the global reference frame and are also represented in Fig. 2. Equation (1) can be used to transform the fields in direction cosines with respect to the global reference frame. Next, Table 3 gives the respective wavelength $\lambda$ and filter transmission $\tau_{\text {filter }}$ of each channel. In this exercise, the mirrors are assumed to have a transmission of 1 ; hence, the system transmission $\tau$ is the product of $\tau_{\text {filter }}$ by the transmission of the butcher block's AR coatings. Finally, for an input beam of irradiance $I_{\text {input }}$ (expressed as $\mathrm{W} / \mathrm{mm}^{2}$ ) on plane $X Y$, the power of the image-forming beam at detector is given by Eq. (2). In this equation, $S=4766.8 \mathrm{~mm}^{2}$ is the area of the entrance pupil projected on plane $X Y$, and $\theta$ is the field elevation. The elevation $\theta$ is between $0.797 \mathrm{deg}$ and $7.290 \mathrm{deg}$ therefore $\cos (\theta) \approx 1$, consequently the power expressed in $\mathrm{W}$ is $P_{\text {det }} \approx I_{\text {input }} \times 4766.8 \tau$. On a detector with $13-\mu \mathrm{m}$ pixels and because the system has a sub-pixel spot size, a single field beam would thus give an irradiance of $I_{\text {input }} \times 2.81 \times 10^{7} \tau \mathrm{W} / \mathrm{mm}^{2}$ on the detector.

$$
\begin{gathered}
\left\{\begin{array}{l}
C=\frac{1}{\sqrt{1+\tan ^{2}\left(f_{x}\right)+\tan ^{2}\left(f_{y}\right)}}, \\
B=C \cdot \tan \left(f_{y}\right) \\
A=C \cdot \tan \left(f_{x}\right)
\end{array},\right. \\
P_{\mathrm{det}}=\frac{I_{\text {input }} \cdot S}{\cos (\theta)} \cdot \tau .
\end{gathered}
$$

This paper starts by describing how the TMA mechanical design is performed to control straight shots and first-order scattering from non-optical surfaces. Qualitative analysis is conducted by performing ray tracings at order zero (i.e., neglecting scatter or specular splits of rays) to verify if any objects is simultaneously illuminated and visible from the detector. The quantitative assessment of the stray light performance requires the identification of the paths foreseen to be the most critical. This contrasts with the brute force approach where rays are traced and every possible interaction in the system is computed. This would be both highly inefficient and hard to interpret. The paths identified are in particular the scattering on the aperture stop, the scattering on the mirrors, and the ghost reflections inside the butcher block. Each contributor is investigated separately by taking advantage of ray tracing software capabilities that allow setting the ray trace properties differently on each surface of the design, therefore considering only certain interactions at a time. In this paper, the analysis is done with the FRED software. ${ }^{12}$ This paper provides general guidelines for control and analysis of stray light in off-axis TMAs. An emphasis is put on design best practices and on the philosophy behind the analysis. This could serve as a recipe for the development of future instruments. The quantitative results extracted from these analyses will be used by the system engineer in the global instrument performance budget, which is out of the scope of this paper. 


\section{Mechanical Structure}

\subsection{Principles and Qualitative Analysis}

The purpose of the mechanical structure is to hold the optical elements and to enclose the system from the outside. It should not affect the image-forming beam (i.e., no vignetting) and it should be optimized for stray light control. Usually, the mechanical surfaces are covered with a black treatment that absorbs most of the light and scatters the residual in all directions. The stray light control is performed by emphasizing on first-order scattering minimization. Scattering paths at second order (i.e., rays undergoing two scattering events) or higher are often neglected as their power are attenuated by the multiple scattering events. Therefore, we should focus on minimizing the number of surfaces that are simultaneously illuminated and seen from the detector as these are the only ones producing first-order stray light. ${ }^{6}$ By definition, surfaces seen from the detector are called critical surfaces. ${ }^{8}$ In a transmission on-axis systems, where rays are forced to follow the natural sequence of elements, no surface located before the aperture stop is critical. ${ }^{8}$ Hence, even if they are illuminated, they cannot produce first-order scattering. Similarly, no surface located after the aperture stop is illuminated by in-field sources. However, it is possible that these surfaces are illuminated by out-of-field sources. ${ }^{8}$ Out-of-field illumination of surfaces after the aperture stop can thus create stray light, which can be controlled by the appropriate use of baffles, apertures, or vanes.

The case of an off-axis TMA is different. First, it is a system in reflection and therefore the aperture stop is simultaneously critical and illuminated. ${ }^{6}$ Second, the off-axis allows unwanted light to propagate without following the natural sequence of elements. This offers the possibility for surfaces located before the aperture stop to be critical or for straight shots to occur. Figure 3 shows a 3D view of the TMA opto-mechanical design, optimized for stray light control. It is composed of the following main elements: the housing, a front-baffle, an aperture labeled $\Pi$, an aperture stop covered with light traps, an FPA baffle, and a mount covered with vanes for the fold mirror. Hereafter, we describe the principles behind the different elements. Figure 4(a) shows the ray tracing of the system with the central fields of channels $\mathrm{R}$ and $\mathrm{T}$.

A front baffle is placed at the entrance of the instrument. It has an input port through which light enters and an exit port in front of M1. Close to the exit port, the bottom surface of the baffle is opened so that the image-forming beam reflected on M1 can reach M2. The input and exit ports enclose the image-forming beam envelope and are asymmetrical due to the shape of the FOV. The front baffle acts as a shield preventing out-of-field light from entering inside the instrument. The longer the baffle compared to its diameter, the more out-of-field light it blocks. Finally, vanes inside the baffle are used to block scattering on its surface. For example, they

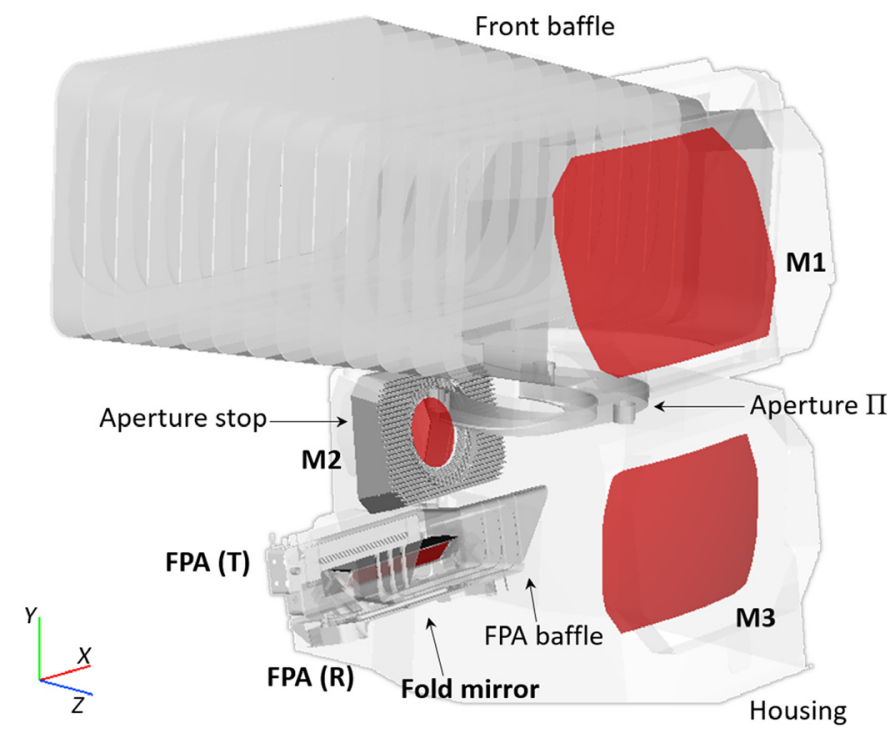

Fig. 3 3D view of the TMA opto-mechanical design. 


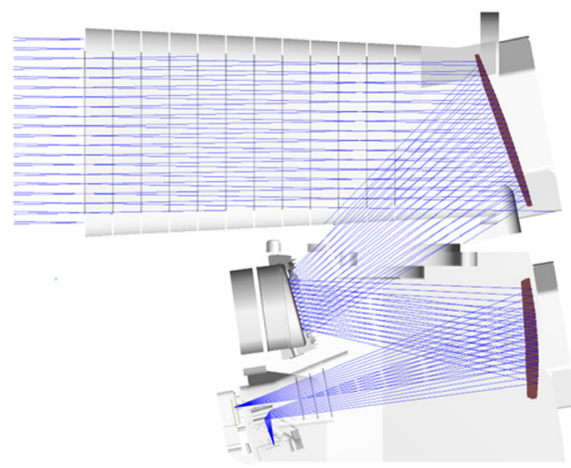

(a)

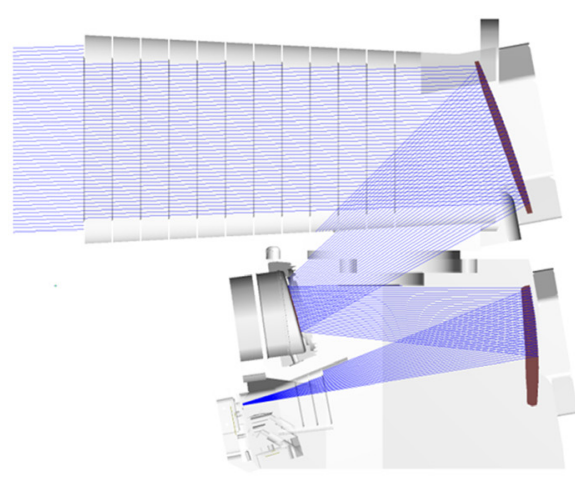

(c)

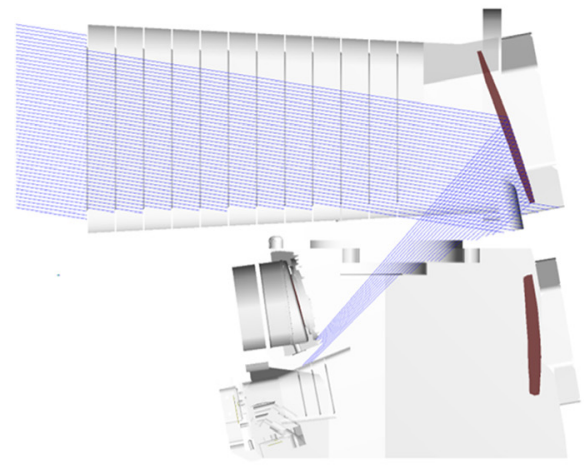

(b)

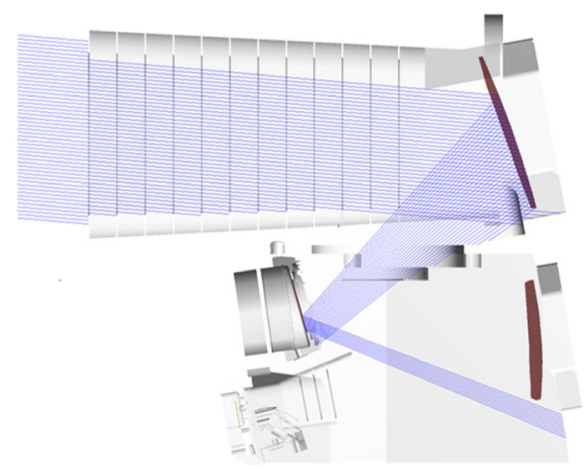

(d)

Fig. 4 (a) Ray tracing of central fields for channels R and T. (b) An out-of-field straight shot at $\left(f_{x}\right.$; $\left.f_{y}\right)=(0 \mathrm{deg} ;-9 \mathrm{deg})$ is blocked by the FPA baffle. (c) Out-of-field light at $\left(f_{x} ; f_{y}\right)=(0 \mathrm{deg} ; 2 \mathrm{deg})$ blocked by internal vanes inside the FPA baffle. (d) Out-of-field light at $\left(f_{x} ; f_{y}\right)=(0 \mathrm{deg} ;-5 \mathrm{deg})$ hitting the housing below M3.

can be placed in a cold shield configuration, ${ }^{6,8,13}$ where no first-order scattering is able to reach the exit port. Next, light reflected on M1 must pass through the aperture $\Pi$ to enter inside the system. This aperture encloses the image-forming beam, blocks part of the unwanted light reflected on M1 (similarly as a diaphragm in an on-axis system), and avoids direct light path toward M3. This includes out-of-field light but also light inside the FOV that is not part of the image-forming beam. The closer the aperture $\Pi$ is from the M2, the more unwanted light is blocked. The front baffle together with aperture $\Pi$ is fundamental in blocking a large part of useless light outside of the system.

The FPA baffle is a baffle placed close to the FPA (cf. Fig. 3), which restricts the direct view from the sensors toward M3. As it hides the direct view toward aperture $\Pi$, it blocks the straight shot reflected on M1 toward the FPA, as shown in Fig. 4(b). With the FPA baffle, the only critical surfaces are the inside of the FPA baffle, the fold mirror mount, and the aperture stop. Vanes are used inside of the FPA baffle, as this one is illuminated by out-of-field sources located close to the FOV [Fig 4(c)]. As the illumination is restricted within a small angular range, only a few vanes are sufficient. Finally, the length of the FPA baffle is kept short to prevent its top surface from being critical through reflection on M3, as this area is necessarily illuminated.

Regarding the housing, the bottom surface below M3 is illuminated by out-of-field stray light [Fig 4(d)] but does not contribute to first-order stray light as it is not a critical area. If this area had been critical, we could have removed that path by increasing the length of the front baffle. Another solution is overdimensioning M3 so that out-of-field light is specularly reflected toward the FPA baffle, instead of scattered toward the sensor.

At intermediary angles between the fields of channels $\mathrm{R}$ and $\mathrm{T}$, light hits the top surface of the fold mirror mount. For channel T, stray light from this surface is avoided by covering it with Vgroove vanes, as shown in Fig. 5. Therefore, the stray light is limited to the very small area of the 


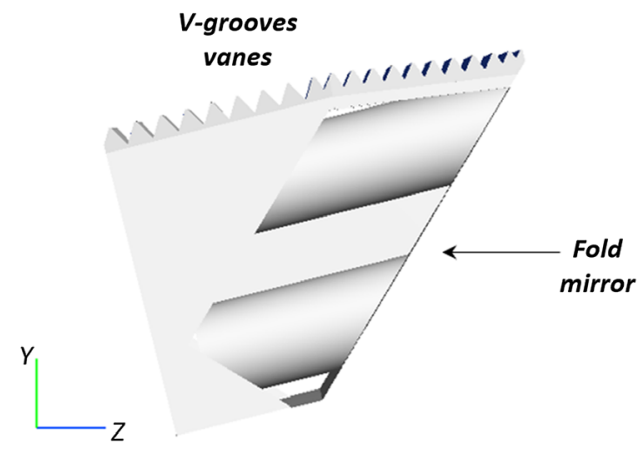

Fig. 5 Side view of the fold mirror mount.

vanes edges. For channel R, a small part of the out-of-field light is able to reach the sides of the mount around the fold mirror effective area. This is also a critical surface and thus the mirror can be slightly overdimensioned to reflect specularly this light away from the sensor.

The aperture stop, surrounding M2, is critical and illuminated by light from both inside and outside of the FOV. Even with an excellent black treatment, a simple flat surface for the aperture stop would lead to a significant stray light. Consequently, a special design for the aperture stop was implemented with V-grooves vanes as shown in Fig. 6(a). The principle of this design is that the top surfaces of the vanes are illuminated but are not seen from the detector; hence, they do not create first-order stray light paths. Analogously, the bottom surfaces of the vanes are seen from the detector but they are not illuminated [Fig 6(b)]. With this design, only the thin edges of the vanes are simultaneously illuminated and critical, thus contributing to first-order stray light. Here, V-groove vanes have rounded edges with thickness $60 \mu \mathrm{m}$.

When designing the V-groove vanes, the angle of their top and bottom planes should be adjusted so that they are greater than the extreme rays angle on the aperture stop, when tracing rays, respectively, from the scene or from the detector. The optimal angle varies as a function of the height $Y$ on the aperture stop; however, it is practical for manufacturing concerns to take the worst case (here, the angle between both sides is $29 \mathrm{deg}$ ). While some margin can be taken on the angle, it should not be too much acute as this increases the total number of vanes, therefore increasing the combined area of the edges.

In our case, a multi-level system of V-groove vanes is used. This is because the vanes could not be placed at the same height as the mirror. Consequently, side surfaces between the different levels are simultaneously critical and illuminated. Yet, we will show that they have a small contribution due to small view factor and because the path involves weak backscattering. Finally, a small ring between the aperture stop and the mirror is also critical and illuminated.

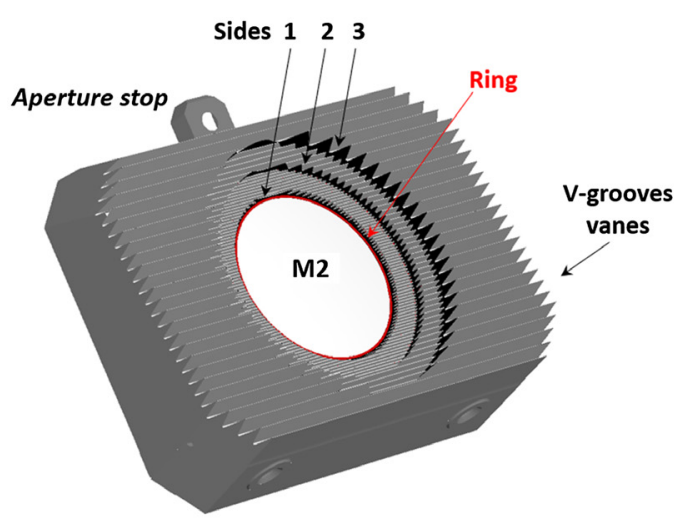

(a)

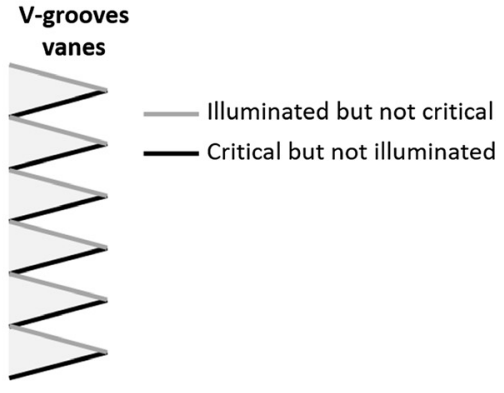

(b)

Fig. 6 (a) Light trap configuration at the aperture stop, made of V-groove vanes on three levels. (b) The top surfaces of the V-groove vanes are illuminated but not critical, whereas the bottom surfaces are critical but not illuminated. 
In summary, the opto-mechanical design of the TMA blocks straight shots and provides stray light control for first-order scattering from every contributor except two. The two exceptions consist of some small components on the aperture stop and the edges of the vanes on the fold mirror mount. This was verified by comparing the surfaces on which rays fall when performing a forward and backward ray tracing with interactions only at zeroth order. The next step is to decide what type of black treatment to apply on the different components. Currently, several black coatings with excellent bidirectional scattering distribution function (BSDF) performances are available on the market. Among them, Acktar black magic, ${ }^{14}$ commonly used in the space industry, is selected for the aperture stop as it can contribute to first-order stray light, with a large quantity of light illuminating it. Surfaces in close proximity with the sensors are also recovered with Acktar (fold mirror mount and FPA baffle). All other components of the design, where first-order scattering is controlled, are recovered with black anodization, which have worse performances than Acktar but present the advantage to be more affordable. The next step is to perform a quantitative analysis to evaluate the impact of the first-order scattering paths. The impact of the vanes edges at the foldmirror mount is neglected as they have a small exposed area, a small quantity of light falling on them, and an excellent black treatment. In the case of the aperture stop, the quantitative analysis is performed at first order as well as at second order as a large quantity of light falls on it.

\subsection{Aperture Stop Quantitative Analysis}

A quantitative analysis is performed to evaluate the impact of scattering on the aperture stop. For that, the point source transmittance (PST) is computed as a function of the field. Defined by Eq. (3), the PST corresponds to the stray light irradiance $\left(I_{\mathrm{FPA}}\right)$ when the instrument is illuminated with a collimated beam of unit irradiance ${ }^{8}\left(I_{\text {input }}\right)$. The PST is computed for the reflection and transmission FPAs. The irradiance is measured on an analysis surface placed right in front of the butcher block and covering the different spectral channels. Hence, for each FPA, we get $\mathrm{PST} / \tau$, which can be multiplied by the system transmission $\tau$ (cf. Table 1 ) to get the stray light

Table 1 Properties of the different spectral channels at center and edge of the line sensors.

\begin{tabular}{|c|c|c|c|c|c|c|c|c|}
\hline Channel \# & $x_{0}(\mathrm{~mm})$ & $y_{0}(\mathrm{~mm})$ & $f_{x}(\mathrm{deg})$ & $f_{y}(\mathrm{deg})$ & $\theta(\mathrm{deg})$ & $\lambda(\mathrm{nm})$ & $\tau_{\text {Filter }}$ & $\tau$ \\
\hline \multirow[t]{2}{*}{ T1 } & 0 & 0 & 0.000 & 1.191 & 1.191 & 482 & 0.70 & 0.69 \\
\hline & 39 & 0 & 7.194 & 1.190 & 7.290 & & & \\
\hline \multirow[t]{2}{*}{ T2 } & 0 & -0.72 & 0.000 & 1.059 & 1.059 & 590 & 0.50 & 0.49 \\
\hline & 39 & -0.72 & 7.192 & 1.059 & 7.268 & & & \\
\hline \multirow[t]{2}{*}{ T3 } & 0 & -1.44 & 0.000 & 0.928 & 0.928 & 562 & 0.50 & 0.49 \\
\hline & 39 & -1.44 & 7.190 & 0.928 & 7.248 & & & \\
\hline \multirow[t]{2}{*}{$\mathrm{T} 4$} & 0 & -2.16 & 0.000 & 0.797 & 0.797 & 655 & 0.50 & 0.49 \\
\hline & 39 & -2.16 & 7.188 & 0.796 & 7.231 & & & \\
\hline \multirow[t]{2}{*}{$\mathrm{R} 1$} & 0 & 0 & 0.000 & -0.817 & 0.817 & 865 & 0.97 & 0.96 \\
\hline & 39 & 0 & 7.162 & -0.816 & 7.207 & & & \\
\hline \multirow[t]{2}{*}{$\mathrm{R} 2$} & 0 & 0.72 & 0.000 & -0.947 & 0.947 & 590 & 0.50 & 0.49 \\
\hline & 39 & 0.72 & 7.160 & -0.946 & 7.221 & & & \\
\hline \multirow[t]{2}{*}{ R3 } & 0 & 1.44 & 0.000 & -1.077 & 1.077 & 443 & 0.97 & 0.95 \\
\hline & 39 & 1.44 & 7.158 & -1.076 & 7.236 & & & \\
\hline \multirow[t]{2}{*}{$\mathrm{R} 4$} & 0 & 2.16 & 0.000 & -1.206 & 1.206 & 590 & 0.50 & 0.49 \\
\hline & 39 & 2.16 & 7.155 & -1.206 & 7.254 & & & \\
\hline
\end{tabular}




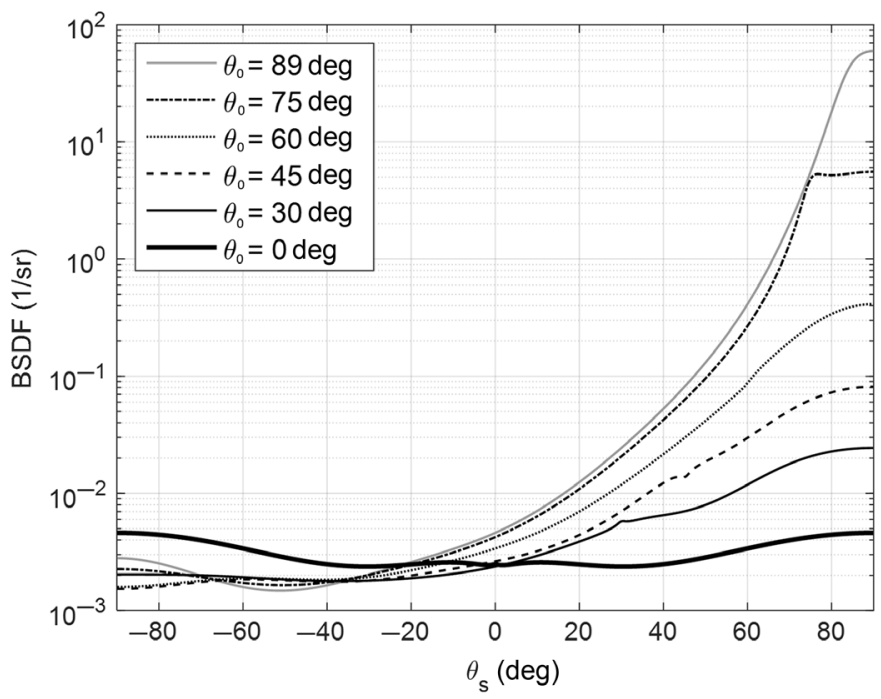

Fig. 7 BSDF of Acktar black magic ${ }^{14}$ as a function of the scatter angle $\theta_{s}$, for different incidence angles $\theta_{0}$. The TIS at normal incidence is $0.93 \%$.

on a given spectral channel. Figure 7 shows the BSDF of Acktar black magic, applied on the aperture stop and used for PST computation. The curves correspond to the average BSDF over the spectral range of the instrument. ${ }^{14}$ While the BSDF angular profile can vary slightly with the wavelength, its total integrated scattering (TIS) stays around 1\%. Here, the TIS is $0.93 \%$ at normal incidence and a scaling of the PST could be performed to evaluate the impact of a different TIS,

$$
\mathrm{PST}=\frac{I_{\mathrm{FPA}}}{I_{\mathrm{input}}}
$$

Stray light is evaluated at first- and second-order scattering. First-order scattering concerns only the edges of the vanes, as well as the sides 1, 2, and 3 as labeled in Fig. 6(a). Importance sampling is used to trace only scattered rays directed toward mirror M3, which limits the ray tracing time by avoiding tracing useless rays. ${ }^{10,11}$ Because the edges of the vanes and sides surfaces are critical directly and not through reflection on $\mathrm{M} 2$, it is not necessary to trace scattered rays toward M2. Indeed, light scattered toward the mirror M2 would not end up at the FPA. The second-order scattering, however, is ray-traced separately by allowing rays to be traced in all directions from any surface on the aperture stop. Figure 8 shows the PST as a function of the field $\left(f_{x} ; f_{y}\right)$, when combining results at first and second orders. As it shows, stray light from the aperture stop occurs mainly for fields inside the instrument FOV. When the field is varied, the footprint of the rays hitting the aperture stop is displaced and gets vignetted for out-of-field sources. Hence, the PST presents some variation inside the FOV and progressively drops to zero outside of the FOV when light is completely blocked by the front baffle or by aperture $\Pi$. An even faster reduction of the out-of-field PST could be achieved by increasing the length for the front baffle.

Figure 9 shows the profile of the PST as a function of $f_{x}$, for $f_{y}=0$. It shows first order, second order, and the decomposition of different paths from first order. As it shows, second order is small compared to first order, with a proportion between $10 \%$ and $17 \%$ of the total stray light. About half of first-order stray light comes from scattering on the edges of the vanes. Hence reducing the thickness of the edges would have a significant impact on the aperture stop stray light, nevertheless it should not be so thin that the black treatment does not adhere. The second contributor to first-order scattering is the ring around M2. Its edges also contribute for a small part of the stray light. Despite the relatively large area of side 1, it has a small view factor and is illuminated at razing incidence; hence, it gives a small backscattering stray light. The view factor for sides 2 and 3 is even smaller, hence they account for a negligible proportion of the stray light and are thus not represented on the graph. 


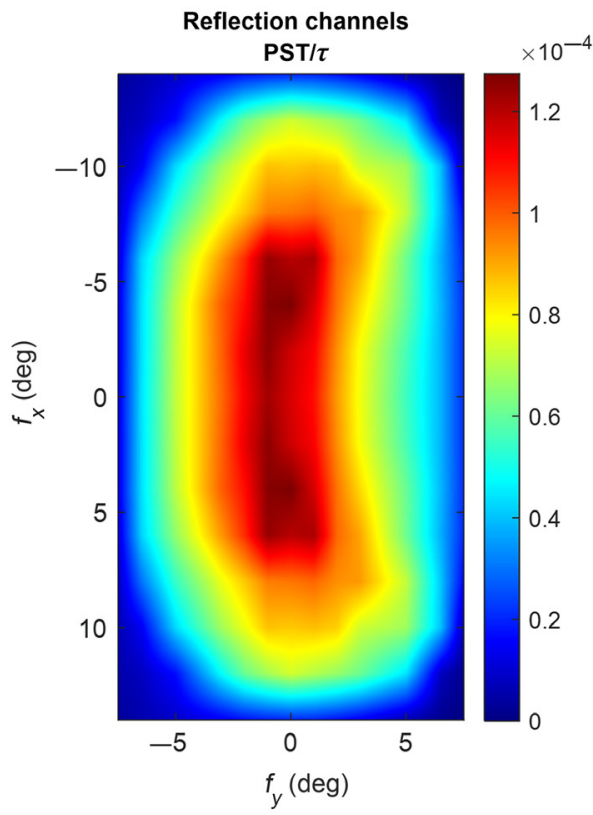

(a)

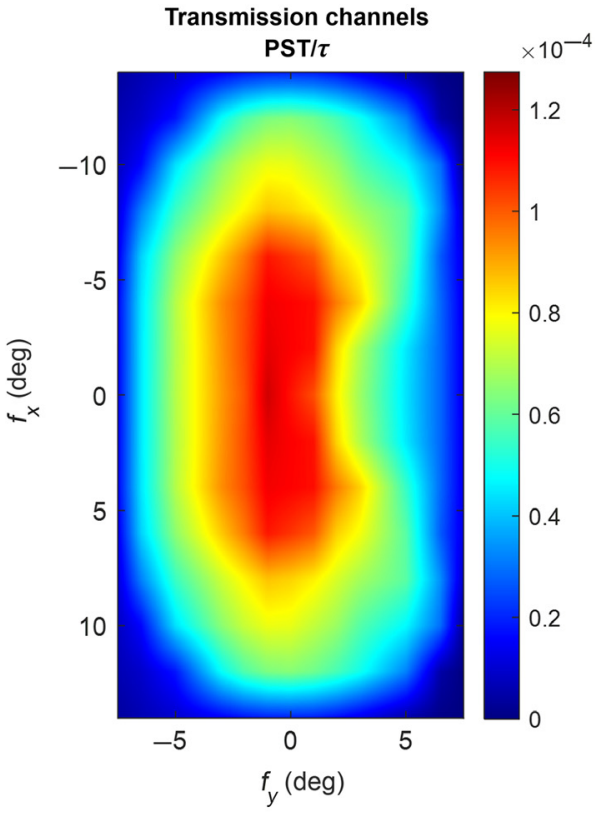

(b)

Fig. 8 PST for first- and second-order scattering on the pupil stop as a function of the field, for (a) reflection and (b) transmission channels.

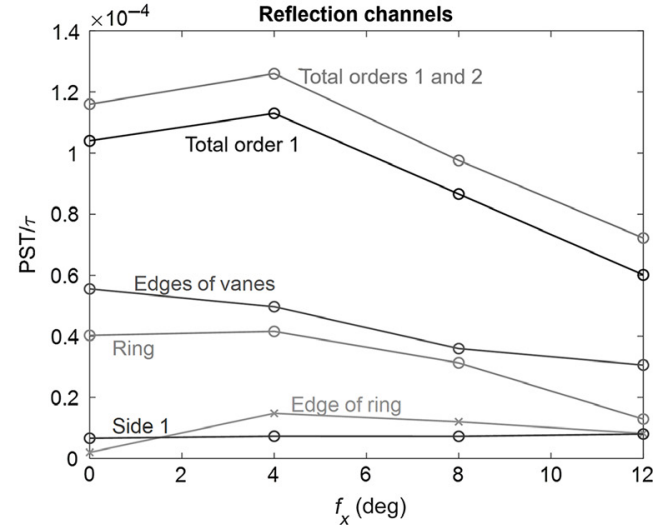

(a)

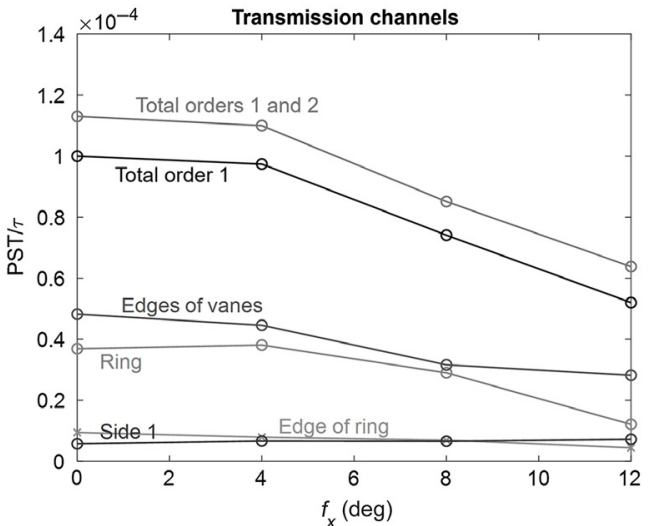

(b)

Fig. 9 Aperture stop PST $/ \tau$ as a function of $f_{x}$, for $f_{y}=0$, decomposed between order 2 scattering and the different paths at order 1 .

\section{Scattering from the Mirrors}

\subsection{Bidirectional Scattering Distribution Function}

The mirrors of the TMA are manufactured by diamond turning followed by polishing and the limitations of the process create roughness on their surfaces. Consequently, light reflected on a mirror is partly scattered away from the specular direction, therefore broadening the PSF. ${ }^{6}$ We are interested in estimating how the scattering on each mirror broadens the nominal spot.

The surface roughness was verified on a flat sample, manufactured with the same process as the TMA mirrors. Figure 10 shows its roughness topology at three different positions, obtained by white light interferometry with zoom $40 \times$. The mean and RMS roughness are given in Table 2; polishing steps were performed until RMS roughness was below $1 \mathrm{~nm}$. To simulate the effect on the instrument, it is important then to derive the BSDF of the as built mirrors. 


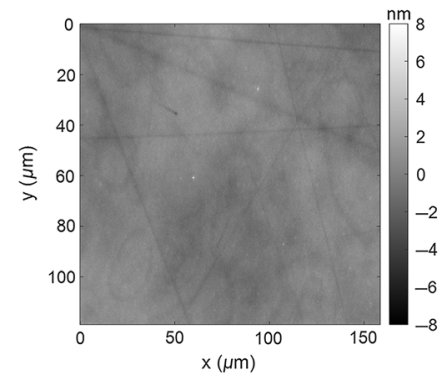

(a)

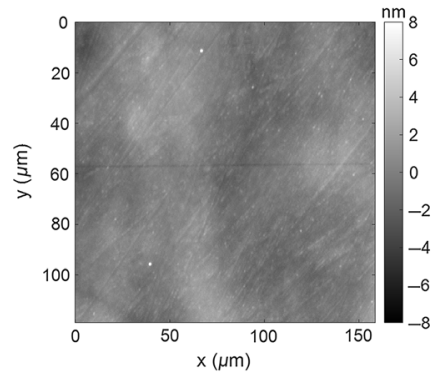

(b)

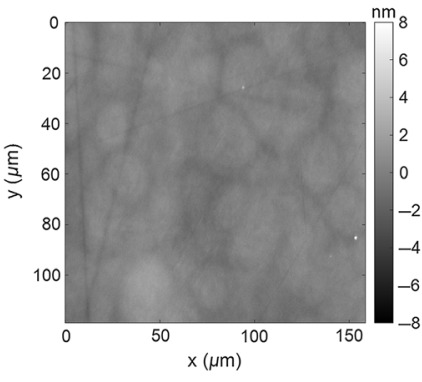

(c)

Fig. 10 Topology map of the mirror measured at different positions of the sample with white light interferometry (zoom 40x).

Table 2 Statistics of the mirror roughness for the topology measurements of Fig 10.

\begin{tabular}{lcc}
\hline \hline Measurement \# & Mean roughness $(\mathrm{nm})$ & RMS roughness $(\mathrm{nm})$ \\
\hline$a$ & 0.534 & 0.652 \\
$b$ & 0.805 & 0.999 \\
$c$ & 0.449 & 0.575 \\
\hline \hline
\end{tabular}

While it is theoretically possible to derive the BSDF from topology measurements, ${ }^{6,15}$ this is complex and model validity is uncertain. Instead, BSDF measurements were directly performed at the facility of ESTEC (European Space Research and Technology Center, ESA), where a sample is illuminated at an incident angle $\theta_{0}$ and the scattered light is measured as a function of the scatter angle $\theta_{s}$, inside the plane defined by the normal and the incident light. Measurements were performed at $633 \mathrm{~nm}$ for different incidence angles. The signature of the BSDF setup was measured as well, it presents a significant signal up to about $\pm 0.045 \mathrm{deg}$ from specular. Above that angle, the signature is negligible compared to the BSDF measured for the sample. Data contained within 0.045 deg from specular were thus removed from the BSDF measurements. Figure 11(a) shows the resulting BSDF curve, with as expected a peak centered on the specular direction and a rapid decrease away from that angle.

A rough optical surface can be modeled with a Harvey model, ${ }^{16}$ whose profile is given by Eq. (4) and depends on three parameters $(b, s$, and $L)$. The BDSF is traditionally plotted as a

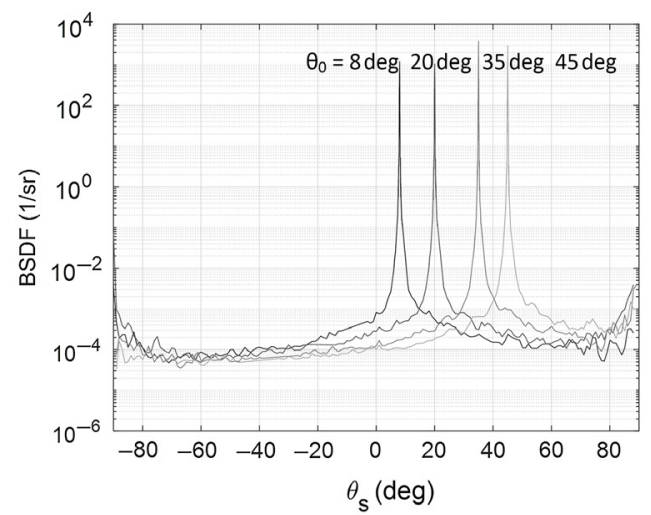

(a)

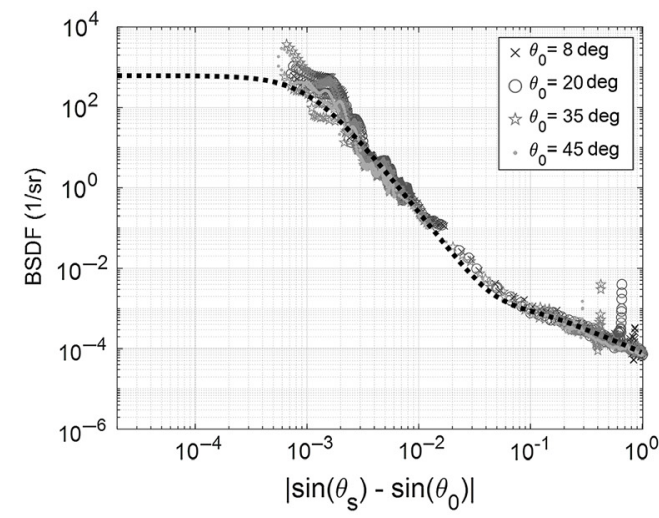

(b)

Fig. 11 (a) Experimental BSDF at $633 \mathrm{~nm}$ measured at different incidence angles. (b) Experimental BSDF on a double log scale, with data close to the specular direction removed, and compared to a double Harvey model fit (fit\#1 from Table 3). 
function of $\left|\sin \theta_{s}-\sin \theta_{0}\right|$ on a double logarithmic scale, ${ }^{6,15}$ emphasizing two distinct regimes: a flat top at the origin and a linearly decreasing behavior at angles further away from specular. Parameter $b$ corresponds to the amplitude of the flat top, $s$ is the slope of the linear regime, and $L$ gives the transition position between the two regimes. ${ }^{6}$ In practice, Fig. 11(b) shows that the experimental data present two linearly decreasing regimes with different slopes. This could be because the roughness profile contains two different roughness at different spatial frequencies. Hence, a fit was performed with a double Harvey model [dotted line in Fig. 11(b)]. In addition, it can be seen that the BSDF increases far away from the specular angle. This is typically a behavior that could have been modeled with an extended version of the Harvey model; nevertheless, this was neglected as it is so far from the specular direction.

$$
\operatorname{BSDF}\left(\theta_{s}, \theta_{0}\right)=b \cdot\left[1+\left(\frac{\left|\sin \theta_{s}-\sin \theta_{0}\right|}{L}\right)^{2}\right]^{s / 2} .
$$

While all simulations in this paper were performed with FRED, the double Harvey model fit was performed with the ASAP software, ${ }^{17}$ which has a built-in tool for that purpose. Table 3 shows different sets of parameters possible for the fit, also plotted in Fig. 12(a). The first Harvey model fits the regime close to specular direction, whereas the second fits the linear regime further away. Different possibilities for the first Harvey model come from the fact that experimental data are only available at $0.045 \mathrm{deg}$ from specular angle. Hence, parameter $b$ representative of the amplitude has the largest relative variation, whereas parameters $s$ and $L$ stay relatively similar. The second Harvey model gives a slop parameter " $s$ " with nearly the same value for each fit. Values for $b$ and $L$ can vary widely between the different fits as they only impact the BSDF at small angles, where the first Harvey model is predominant. Equation (5) can then be used to compute the TIS. As shown in Table 3, the first Harvey model gives a TIS around $0.41 \%$ with a relative variation of $19.4 \%$ between the different fits. The second Harvey model presents a TIS of $0.06 \%$ with negligible variation between the different fit results. For correlation purposes, we can use Eq. (5) to compute an effective roughness value for each model. This approach is not fully representative of the real roughness of the mirror, yet it is in order of magnitude similar to the actual measured roughness, with a value around 3.23 and $1.18 \mathrm{~nm}$, respectively, for the first and second Harvey models.

In this paper, we use the parameters from fit result \#1 for simulating the impact of mirrors roughness on the instrument performance. The BSDF at any wavelength can be deducted by scaling the parameters with the wavelength as per the Harvey model formalism ${ }^{6}\left(b \propto 1 / \lambda^{4}\right.$, $s=$ constant, $L \propto \lambda$ ). Figure 12(b) shows the different Harvey model fits scaled at 400 and $800 \mathrm{~nm}$. As shown in Fig. 12(c) and by Eq. (5), the TIS decreases with the wavelength and thus the impact on the instrument is the worse at short wavelengths, ${ }^{6}$

Table 3 Different fit results with a double Harvey model.

\begin{tabular}{|c|c|c|c|c|c|c|c|c|c|c|}
\hline \multirow{2}{*}{$\frac{\lambda=633 \mathrm{~nm}}{\text { Fit \# }}$} & \multicolumn{5}{|c|}{ Harvey 1} & \multicolumn{5}{|c|}{ Harvey 2} \\
\hline & $b$ & $S$ & $L$ & TIS (\%) & $\sigma_{\text {eff }}(n m)$ & $b$ & $s$ & $L$ & TIS (\%) & $\sigma_{\text {eff }}(\mathrm{nm})$ \\
\hline 1 & 1421.78 & $3-3.92214$ & 0.00101 & 0.48 & 3.48 & 0.121248 & -1.10956 & 0.0014 & 0.06 & 1.20 \\
\hline 2 & 615.90 & -3.46354 & 1.05E-03 & 0.29 & 2.73 & 0.00113 & -1.20090 & $1.11 \mathrm{E}-01$ & 0.05 & 1.15 \\
\hline 3 & 804.95 & -3.75544 & 1.10E-03 & 0.35 & 2.98 & 0.09863 & -1.04348 & $1.11 \mathrm{E}-03$ & 0.05 & 1.17 \\
\hline 4 & 2113.29 & -3.59878 & 7.73E-04 & 0.50 & 3.55 & 28.04790 & -1.07004 & $6.62 \mathrm{E}-06$ & 0.05 & 1.18 \\
\hline 5 & 1109.49 & -3.78642 & $1.02 \mathrm{E}-03$ & 0.40 & 3.21 & 1.10837 & -1.16382 & 2.70E-04 & 0.06 & 1.22 \\
\hline 6 & 1508.07 & -3.79330 & $9.41 \mathrm{E}-04$ & 0.47 & 3.45 & 611.84 & -1.07140 & 3.83E-07 & 0.06 & 1.18 \\
\hline Mean & 1262.25 & -3.71994 & 0.00098 & 0.41 & 3.23 & 106.87 & -1.10987 & 0.01888 & 0.06 & 1.18 \\
\hline STD/mean & $42.8 \%$ & $4.4 \%$ & $11.8 \%$ & $19.4 \%$ & $10.0 \%$ & $231.7 \%$ & $5.5 \%$ & $237.8 \%$ & $3.8 \%$ & $1.9 \%$ \\
\hline
\end{tabular}




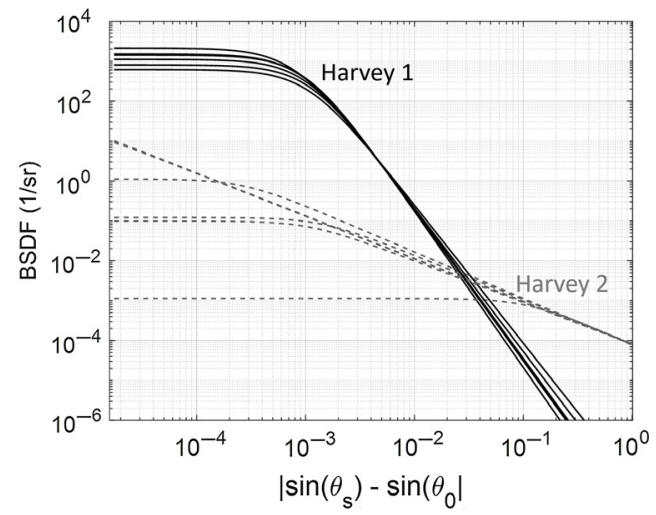

(a)

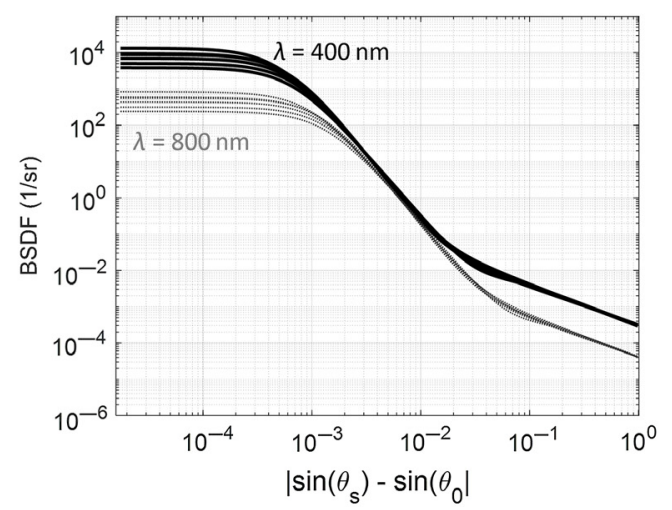

(b)

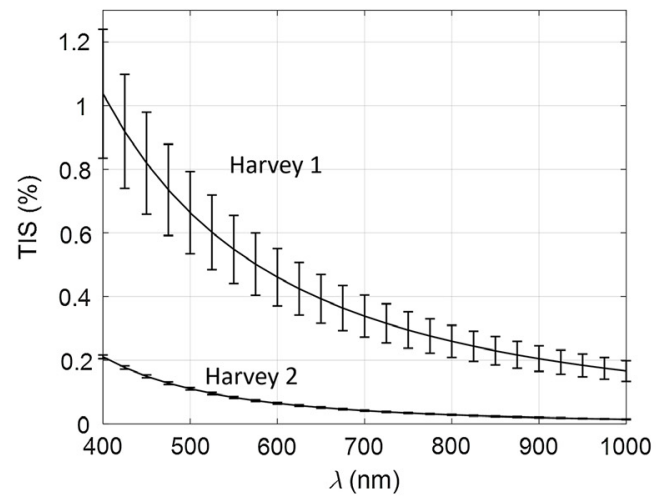

(c)

Fig. 12 (a) Harvey models with the parameters from Table 3 at $633 \mathrm{~nm}$. (b) Spectral scaling of the double Harvey model from Table 3, at 400 and $800 \mathrm{~nm}$. (c) TIS as a function of the wavelength for the two Harvey models.

$$
\mathrm{TIS}=\frac{2 \pi b}{L^{s}(s+2)} \cdot\left[\left(1+L^{2}\right)^{\frac{s+2}{2}}-\left(L^{2}\right)^{\frac{s+2}{2}}\right]=\left(\frac{4 \pi \sigma_{\mathrm{eff}}}{\lambda}\right)^{2}
$$

\subsection{Ray Tracing Results}

The BSDF was introduced in the ray-tracing model and first-order scattering was computed for each mirror separately. The spatial point source transmittance (SPST) is obtained by measuring the stray light irradiance map at the detector for an input collimated beam of unit irradiance in $X Y$. SPST and PST share the same definition but the SPST contains spatial information while PST is an average over the detector. The SPST is useful in situations, such as optical surface scattering, where stray light is not uniformly distributed. Figure 13 shows the SPST maps, normalized to instrument transmission $\tau$, for scattering on mirrors M1 (a), M2 (b), and M3 (c) considering the center of field for channel T3. The SPST maps are centered on the position of the nominal spot, $\left(x_{0} ; y_{0}\right)$, and have a symmetrically decreasing profile when departing from the center. The profile along the $x$ direction on the line sensor is shown in Fig. 13(d) for each mirror. The sum of the contribution from each mirror gives the total scattering SPST map. Scattering on M2 gives an SPST with the largest amplitude while M1 gives the weakest. The full width at half maximum (FWHM) follows the opposite trend, with an SPST profile the larger for M1 and the thinner for M2. The reason for that is that light scattered on M2 converges closer to the nominal spot as it is reflected on convex mirror M3 while light scattered on M1 is brought further apart due to reflection on concave mirror M2. The stray light power is thus concentrated on a smaller area for M2.

The SPST is to be compared with the nominal irradiance for unit input irradiance, which for a pixel of $13 \mu \mathrm{m}$ is $2.81 \times 10^{7} \tau \mathrm{W} / \mathrm{mm}^{2}$. The value of the SPST for mirror scattering looks very 


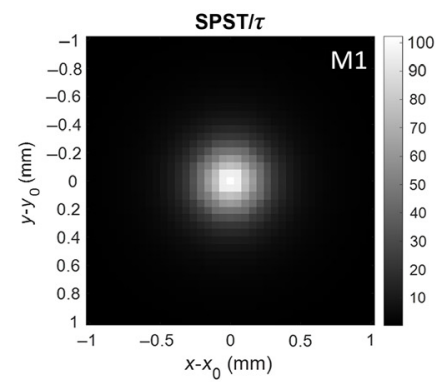

(a)

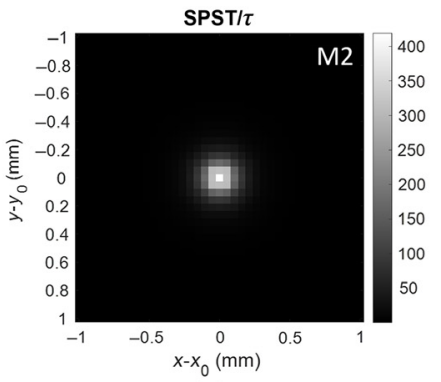

(b)

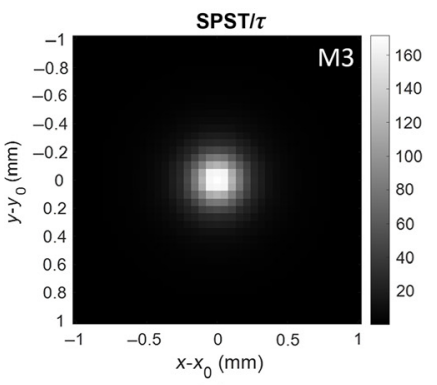

(c)

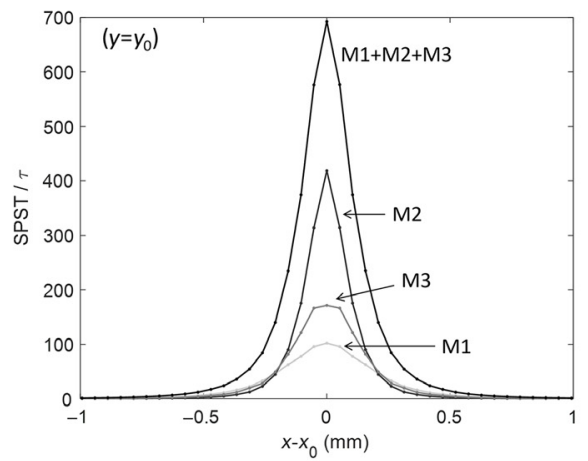

(d)

Fig. 13 SPST maps from scattering on mirrors (a) M1, (b) M2, and (c) M3 and (d) SPST maps radial profiles, for center of channel T3 $\left[\lambda=562 \mathrm{~nm},\left(f_{x} ; f_{y}\right)=(0 ; 0.928 \mathrm{deg})\right]$. In comparison, the sensor length is $\pm 39 \mathrm{~mm}$.

high compared to the PST from the pupil stop. There is however a fundamental difference as the first gives a highly localized stray light pattern while the second gives a stray light signal distributed over the full detector. The first decreases the instrument resolution limit while the second decreases the signal-to-noise ratio.

The SPST maps were computed for the different channels. The maximum value of SPST $/ \tau$ and the FWHM are plotted as a function of the channel wavelength in Figs. 14(a) and 14(b). The value of $\max [\operatorname{SPST} / \tau]$ decreases with the wavelength while the FWHM presents an increasing trend (small deviations are explained by ray tracing stochastic error). This behavior can be explained by the fact that when the wavelength increases, the amplitude $b$ of the BSDF decreases while parameter $L$ increases, therefore broadening the scattering profile.

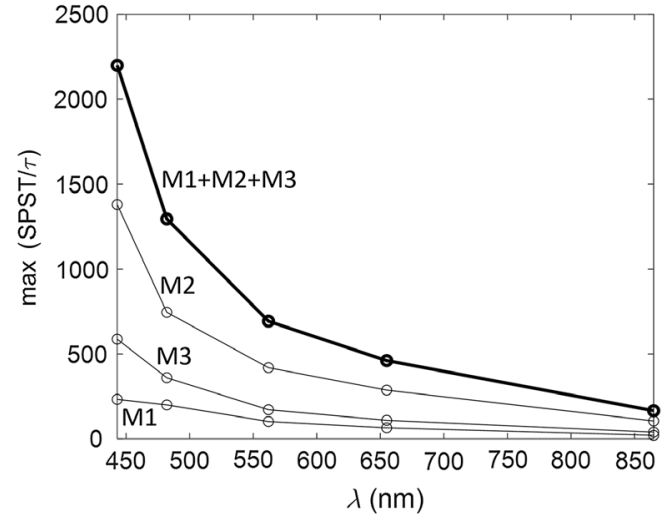

(a)

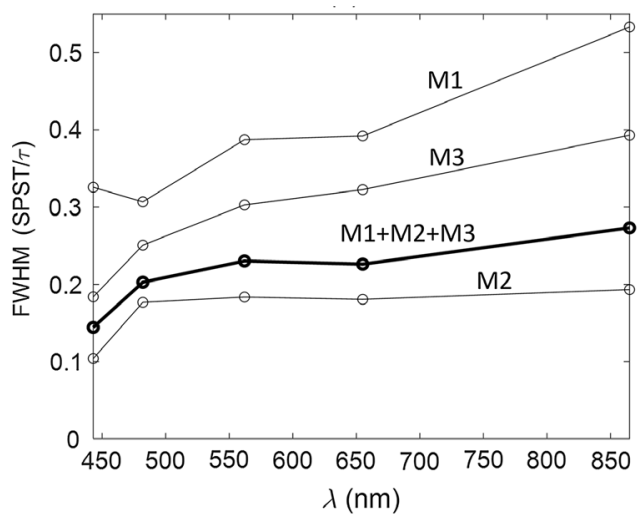

(b)

Fig. 14 (a) Maximum value and (b) FWHM of the SPST maps from scattering on M1, M2, and M3 and combined effect, as a function of the wavelength of the different channels. 


\section{Ghosts from the Butcher Block}

\subsection{Butcher Block Model}

A butcher block is placed in front of each FPA. It holds four filter stacks placed in front of the line detectors to perform the channel spectral selection [Fig 15(a)]. A black mask isolates the stacks from each other. As illustrated in Fig. 15(b), each filter stack consists of two NZK7 rods enclosing a spectral filter in-between. AR coatings are applied on the input and output interfaces of the stack to minimize ghost reflections. The spectral filters are assumed to have a non-zero transmission only at the wavelength associated to their channel (i.e., no direct cross-talk). At that wavelength, the transmission is given by $\tau_{\text {filter }}<1$ (cf. Table 1) and their reflectivity is $R_{\text {filter }}=1-\tau_{\text {filter }}$. The reflectivity of the AR coating is given in Fig. 16(a) as a function of the wavelength, for various incidence angles by steps of $10 \mathrm{deg}$. The image-forming beam has a maximum incidence angle of about $11 \mathrm{deg}$ and therefore the reflectivity in our model is considered as the maximum reflectivity value between $0 \mathrm{deg}$ and $10 \mathrm{deg}$ incidence angles. As shown in Fig. 15(a), a black mask is placed at the output surface of the filter stack. For incidence angles between $0 \mathrm{deg}$ and $10 \mathrm{deg}$, its internal and external reflectivities are shown as a function of the wavelength in Fig. 16(b). Finally, the detector itself is not perfectly absorbing but reflects about $20 \%$ specularly.

\subsection{Ghost Analysis}

Ghost analysis of the butcher block is performed for different channels. Only the central field $\left(f_{x}=0\right)$ is considered as the optical design is quasi-telecentric. Indeed, at the detector, the chief ray has an angle of incidence slightly above zero in the $y$ direction [as visible in Fig. 15(a)], but it has no angle component in the $x$ direction. Therefore, the fan of rays has the same properties regardless of the field $f_{x}$.

Figure 17(a) shows the ghost SPST map for channel T3. The stray light on the line detector corresponds to the profile along $x$ for $y=y_{0}$, shown in Fig. 17(b). The SPST map is composed of

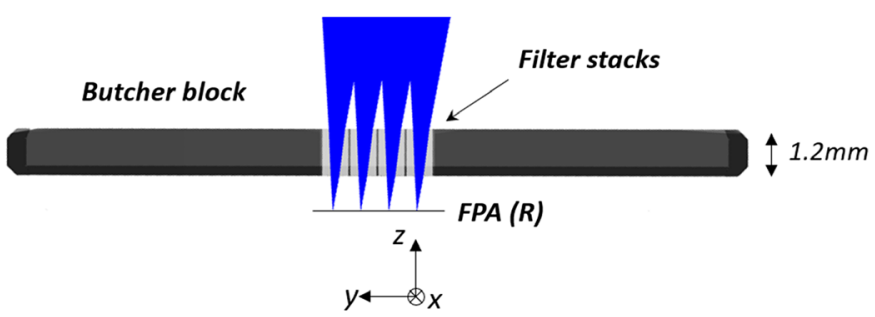

(a)

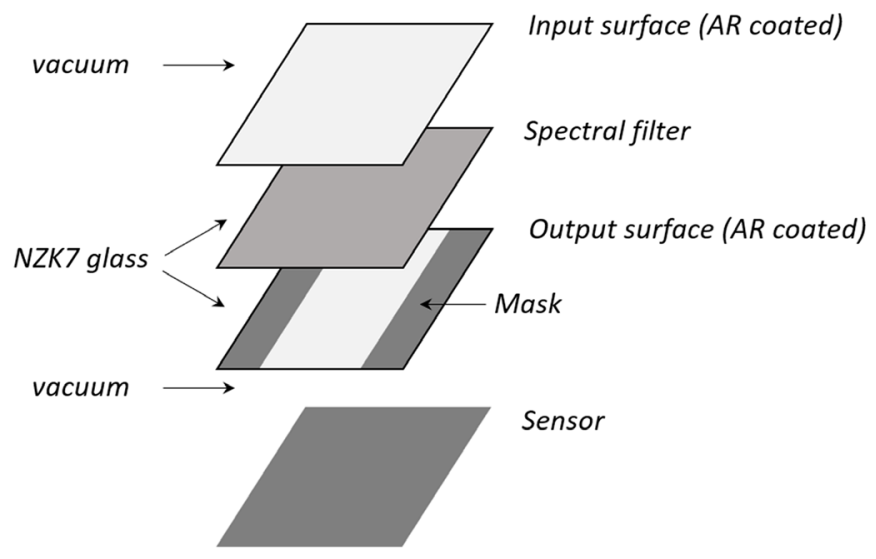

(b)

Fig. 15 (a) Butcher block with four filter stacks placed in front of the line detectors. (b) Sketch of the components of the filter stack for a single channel. 


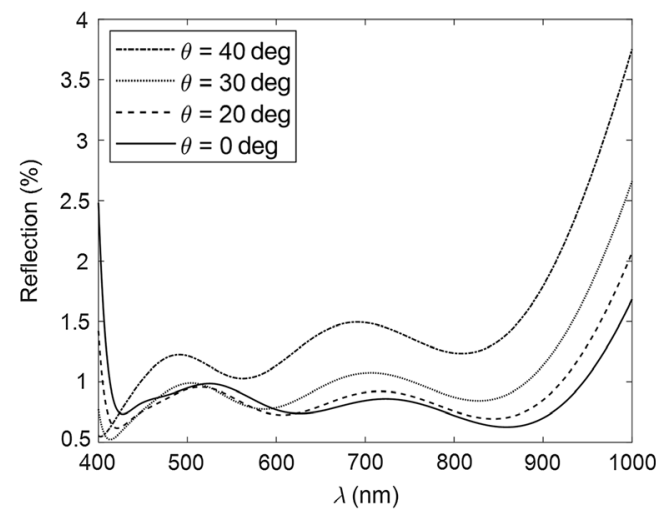

(a)

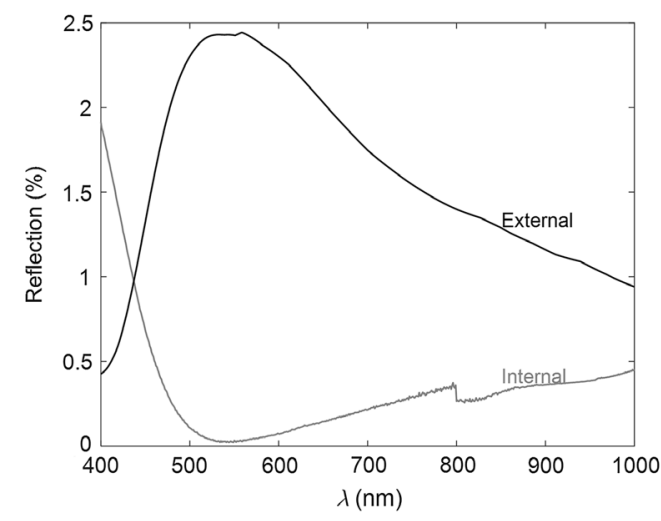

(b)

Fig. 16 (a) Reflection spectrum of the AR coating for different incidence angles. (b) Reflection spectrum of the internal and external surface of the black mask placed at the output surface of the butcher block (maximum value between 0 deg and 10 deg incidence angle). The coatings are developed in-house.

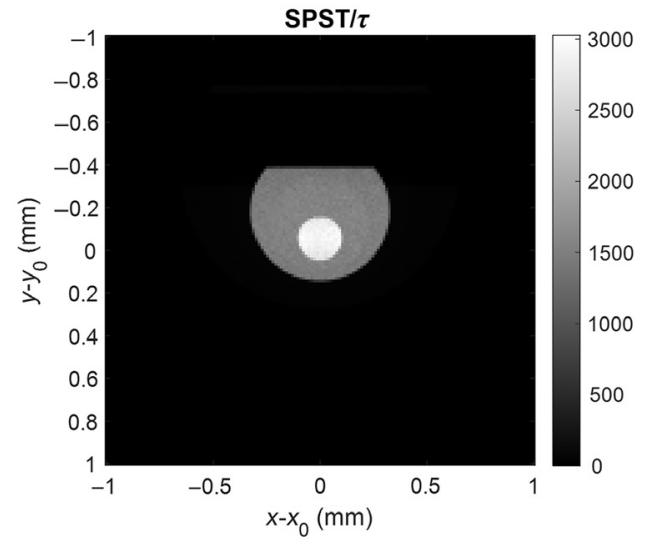

(a)

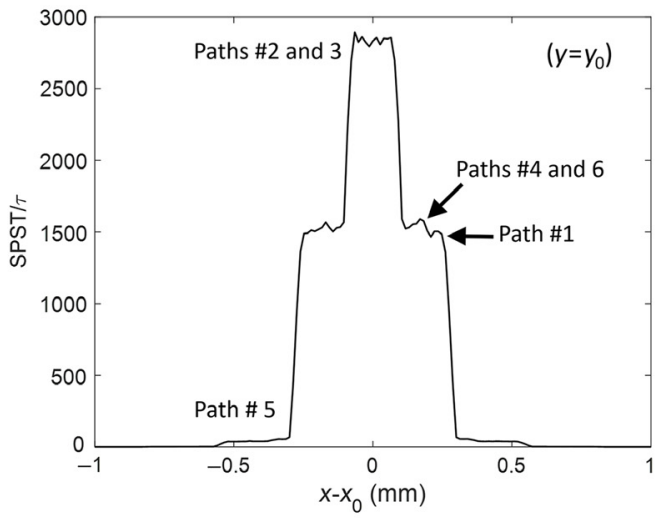

(b)

Fig. 17 (a) SPST maps from ghosts at the butcher block and profile along the $x$ direction (b) for channel T3 $\left[\lambda=562 \mathrm{~nm},\left(f_{x} ; f_{y}\right)=(0 ; 0.928 \mathrm{deg})\right]$. The path indicated on the profile is related to Table 4.

a superposition of ghosts with different sizes and irradiances, giving a profile with three main plateaus. The ghosts are symmetrical with respect to $x$ axis but slightly decentered along $y$ due to the tilt of the fan of rays. A path analysis was performed and Table 4 shows the 2D integrated power at the FPA for the different paths. First, it shows that fourth-order ghosts (ghost rays with four reflections) are negligible in front of second order (ghost rays with two reflections). At second order, the ghost with the highest total power is path \#1 and comes from the reflection between the sensor and the filter, the two surfaces with the higher reflectivities. However, it has a large optical path length so the ghost is largely defocused, hence irradiance on a given pixel is low. In Fig. 17(a), this ghost is the largest clearly visible with a circular shape and partly vignetted by the mask. On the profile, it corresponds to the intermediary plateau. The second highest contributors are the reflections between the filter and either the output (path \#2) or input (path \#3) surfaces of the butcher block. They have a smaller path length and so they are more focused than path \#1. They correspond to the ghost with the smaller radius but the higher irradiance. Paths \#4 and \#6 from Table 4 involve an optical path length slightly smaller than path \#1. They produce ghosts visible as bumps on the intermediary plateau of the profile (path \#6 has a power 
Table 4 Power proportion for the different ghosts occurring at the butcher block.

\begin{tabular}{|c|c|c|c|c|c|c|c|c|}
\hline & \multirow[b]{2}{*}{ Path \# } & \multicolumn{5}{|c|}{ Stray light power/nominal power (integrated) } & \multirow[b]{2}{*}{$\begin{array}{l}\text { First ghost } \\
\text { surface }\end{array}$} & \multirow[b]{2}{*}{$\begin{array}{l}\text { Second ghost } \\
\text { surface }\end{array}$} \\
\hline & & $\begin{array}{c}482 \mathrm{~nm} \\
\mathrm{~T} 1\end{array}$ & $\begin{array}{c}562 \mathrm{~nm} \\
\text { T3 }\end{array}$ & $\begin{array}{c}655 \mathrm{~nm} \\
\mathrm{~T} 4\end{array}$ & $\begin{array}{c}443 \mathrm{~nm} \\
\mathrm{R} 3\end{array}$ & $\begin{array}{c}865 \mathrm{~nm} \\
\mathrm{R} 1\end{array}$ & & \\
\hline \multirow{6}{*}{$\begin{array}{l}\text { Individual paths } \\
\text { at order } 2\end{array}$} & 1 & $5.22 \%$ & $8.71 \%$ & $8.75 \%$ & $0.54 \%$ & $0.53 \%$ & Sensor & Filter \\
\hline & 2 & $0.27 \%$ & $0.45 \%$ & $0.39 \%$ & $0.16 \%$ & $0.13 \%$ & Output surface & Filter \\
\hline & 3 & $0.27 \%$ & $0.45 \%$ & $0.39 \%$ & $0.11 \%$ & $0.08 \%$ & Filter & Input surface \\
\hline & 4 & $0.18 \%$ & $0.18 \%$ & $0.15 \%$ & $0.02 \%$ & $0.02 \%$ & Sensor & Output surface \\
\hline & 5 & $0.06 \%$ & $0.03 \%$ & $0.03 \%$ & $0.02 \%$ & $0.02 \%$ & Sensor & Input surface \\
\hline & 6 & $0.00 \%$ & $0.00 \%$ & $0.00 \%$ & $0.01 \%$ & $0.00 \%$ & Output surface & Input surface \\
\hline Total order 2 & - & $6.00 \%$ & $9.82 \%$ & $9.70 \%$ & $0.86 \%$ & $0.79 \%$ & - & - \\
\hline Total order 4 & - & $0.21 \%$ & $0.56 \%$ & $0.53 \%$ & $0.00 \%$ & $0.00 \%$ & - & - \\
\hline
\end{tabular}

significantly smaller than path \#4 so in practice we only see the latter). Finally, the path \#5 corresponds to a reflection between the sensor and the input surface. It has the largest optical path length and thus is highly defocused. It is visible on the profile with a very small irradiance, and it is nearly invisible on the $2 \mathrm{D}$ map.

Along the $y$ axis, second-order ghosts are comprised within a radius smaller than $0.6 \mathrm{~mm}$ around $y_{0}$. As the different spectral channels have their line detector at $0.72 \mathrm{~mm}$ from each other, second-order ghosts do not create cross-talk between the different channels. Cross-talk could only occur due to higher order ghosts, which as shown in Table 4 is very small. Indeed, not only do they lose a large quantity of power with the multiple ghost reflections that they undergo, they are widely dispersed as their optical path length is large.

\section{Conclusions}

In this paper, the stray light control and analysis method for an off-axis TMA is presented. The mechanical design aims at minimizing first-order scattering and blocks straight shots. A front baffle is used in combination with an aperture $\Pi$ to restrict the quantity of useless light inside the system. Close to the FPA, an FPA baffle restricts the view from the sensors toward M3. This prevents out-of-field straight shots from reaching directly the sensors after reflection on M1, and it limits the critical areas to the aperture stop and the surfaces close to the FPA. As a large quantity of light falls on the aperture stop, its surface is covered with V-groove vanes. This reduces significantly the first-order scattering contribution, occurring only on the edges of the vanes, a small ring around M2 and sides between the multi-levels vanes. The PST is evaluated as a function of the field and it shows that edges have the most significant contribution, whereas secondorder scattering is much smaller. Next, BSDF of mirror roughness was measured and modeled with a double Harvey function. SPST maps are computed for the scattering of the different mirrors. Mirror M2 gives a stray light profile with the highest amplitude but the smallest FWHM. Moreover, the scattering has the worse contribution at the smallest wavelengths. Finally, we evaluated the ghost reflections inside the butcher block. The spectral filter and the sensors are the two surfaces with the higher reflectivities. However, a reflection between them creates a ghost widely spread at the detector and thus a small irradiance. Reflections between input or output surfaces of the stack with the filter give the most focused ghosts. Fourth-order ghosts are negligible compared to second-order ghosts and there is no cross-talk between the different spectral channels. This paper provides a general guideline on the way to control and analyze stray light for off-axis TMAs. Quantitative results are then provided to the system engineer who considers them in the global instrument performance budget. 


\section{Acknowledgments}

This work was performed under the ESA contract Proba-Evo for OIP Sensor Systems.

\section{References}

1. L. G. Cook "Three-mirror anastigmat used off-axis in aperture and field," Proc. SPIE 0183, 207-211 (1979).

2. G. E. Romanova and K. D. Rodionova (Butylkina), "Design and analysis of the mirror system with off-axis field-of-view," Proc. SPIE 10745, 1074514 (2018).

3. J.-U. Lee and S.-M. Yu, "Analytic design procedure of three-mirror telescope corrected for spherical aberration, coma, astigmatism, and petzval field curvature," J. Opt. Soc. Korea 13(2), 184-192 (2009).

4. S. Risse et al., "Novel TMA telescope based on ultra-precise metal mirrors," Proc. SPIE 7010, 701016 (2008).

5. Q. Meng et al., "Design of off-axis three-mirror systems with ultrawide field of view based on an expansion process of surface freeform and field of view," Appl. Opt. 58(3), 609-615 (2019).

6. E. Fest, Stray Light Analysis and Control, SPIE Press, Bellingham, Washington (2013).

7. V. Kirschner, "Stray light analysis and minimization," Unpublished tutorial from Space Optics Instrument Design \& Technology (SOID) Poltu Quatu, (2017).

8. R. P. Breault, "Control of stray light," Chapter 38 in Handbook of Optics, M. Bass et al., Eds., Vol. 1, pp. 38.1-38.35, McGraw-Hill (1995).

9. S. Grabarnik, "Optical design method for minimization of ghost stray light intensity," Appl. Opt. 54(10), 3083-3089 (2015).

10. R. Pfisterer, "FRED optical engineering software tutorial," Unpublished software tutorial manual from Photon Engineering (2014).

11. R. Pfisterer, "Clever tricks in optical engineering," Proc. SPIE 5524, 1-10 (2004).

12. R. Pfisterer, "FRED software," Version 18.61, edited by Photon Engineering, www .photonengr.com (2014).

13. E. R. Freniere, "First-order design of optical baffles," Proc. SPIE 0257 (1981).

14. "Acktar black treatment datasheet," Unpublished document provided by Acktar Ltd., www .acktar.com.

15. J. C. Stover, Optical Scattering: Measurement and Analysis, 3rd ed., SPIE Press, Bellingham, Washington (2012).

16. J. E. Harvey, Understanding Surface Scatter: A Linear Systems Formulation, SPIE Press, Bellingham, Washington (2019).

17. R. Breault, "ASAP software," edited by Breault Research, www.breault.com (2012).

Lionel Clermont is an optical engineer at the Space Center of Liège (CSL), with a background in design and test of space optical instruments. He has contributed to multiple missions for the European Space Agency as well as for NASA or the Indian Space Agency. His experience in stray light concerns control and analysis of space instruments and industrial systems, calibration as well as stray light correction algorithms. He has also taught stray light at the University of Liège and at the Chinese Academy of Space Technology.

Ludovic Aballea is a system engineer at OIP Sensor Systems. He received his $\mathrm{PhD}$ from Lille University of Science and Technology in 2009, where he worked on the development of a terahertz spectrometer. At OIP, he worked on different projects such as Proba-V, NOMAD, and ALTIUS. 